\title{
Medida no invasiva de la presión arterial central mediante tonometría por aplanamiento. Análisis de la onda de pulso
}

\author{
Non-invasive assessment of central blood pressure \\ by applanation tonometry. Pulse wave analysis
}

\begin{abstract}
Álvaro Hermida Ameijeiras; José Enrique López Paz; Carlos Calvo Gómez
Unidad de Hipertensión y Riesgo Vascular. Servicio de Medicina Interna. Hospital Clínico Universitario de Santiago de Compostela (CHUS). SERGAS. Santiago de Compostela.
\end{abstract}

\section{Introducción}

La tonometría por aplanamiento (TA) es una medida indirecta, no invasiva, reproducible y validada para estimar la forma de la onda de pulso aórtica ${ }^{1-3}$. La propia forma de la onda de pulso, junto con la amplitud de la misma o su duración aportan información útil para el diagnóstico y manejo de diferentes entidades nosológicas (ej. cambios asociados al envejecimiento, hipertensión arterial, cardiopatía isquémica, diabetes mellitus, hábito tabáquico, ...). Por este motivo y en virtud de los numerosos estudios clínicos, tanto observacionales como de intervención publicados hasta la fecha, la Sociedad Europea de Hipertensión (ESH) conjuntamente con la Sociedad Europea de Cardiología (ESC), reconocen que "... es posible que un uso clínico más generalizado de las determinaciones de velocidad de la onda de pulso e índice de potenciación añada más exactitud a la evaluación de la lesión arterial..."‥ Además, el interés del análisis de la onda de pulso no radica únicamente en una correcta estimación del riesgo cardiovascular sino también como herramienta útil para el seguimiento de los pacientes o como criterio adicional a la hora de seleccionar una estrategia terapéutica determinada.

Desde la Unidad de Hipertensión Arterial y Riesgo Vascular del Hospital Clínico de Santiago de Compostela, se realiza diariamente un esfuerzo en implementar el análisis de la onda pulso mediante la TA para la evaluación integral del riesgo cardiovascular de nuestros pacientes, junto con otras técnicas validadas en la detección de aterosclerosis subclínica. En este trabajo, se exponen y discuten aquellos aspectos relacionados con la metodología e interpretación de los resultados así como el valor pronóstico de los diversos parámetros obtenidos mediante esta técnica.

\section{Antecedentes históricos}

Si bien los primeros intentos documentados de registrar la forma de la onda de pulso datan de 1860, por el médico e investigador francés Etienne Jules Marey (1830-1904), Figura 1$)^{5}$, no fue hasta principios del siglo XX, con el desarrollo del cateterismo cardíaco a manos del Dr. Werner Forssman (Premio Nobel de Medicina en 1956), cuando se aportaron los primeros datos que correlacionaban las presiones arteriales centrales con la forma de la onda de pulso periférica ${ }^{6}$.

Figura 1. "Esfigmógrafo" diseñado por el Dr. Etienne Jules Marey en 1881 y publicado en "La méthodegraphiquedans les sciences espérimentales et principalement en physiologie et en médicine" en el año 1885.

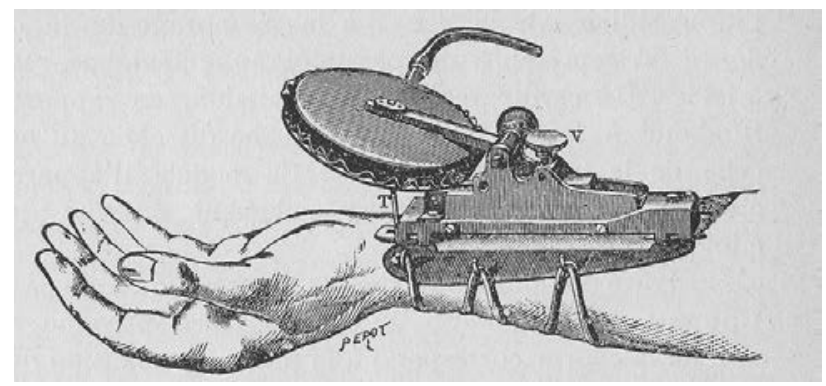

En ese transcurso de tiempo, el desarrollo del primer esfigmomanómetro en 1896 por el médico italiano Scipione Riva-Rocci junto con la incursión del método auscultatorio del médico militar ruso Nicolai-Sergievic Korotkoff en 1905, habían desviado toda la atención de la comunidad científica en la lectura de los valores de presión correspondientes a la pérdida y recuperación del latido arterial, dejando a un lado otros aspectos de la onda de pulso como amplitud de la onda, la pendiente de ascenso/descenso o el estudio de la onda de reflexión.

En 1955, Kroeker y Wood7 objetivaron cómo la forma de la onda de pulso cambiaba a lo largo del árbol vascular como resultado del incremento de la presión arterial sistólica junto con el incremento de las presiones periféricas. Pocos años después, e inspirados en la técnica de tonometría ocular, G.L. Pressman y P. M. Newgard desarrollaron el primer tonómetro arterial ${ }^{8}$. Desde ese momento se desarrollaron diversos dispositivos para el registro y análisis de la onda de pulso y en la actualidad existen comercializados y validados por diversas autoridades gubernamentales en el ámbito sanitario asi como sociedades científicas, distintos modelos, alguno de los cuales permite incluso la monitorización de la 
Figura 2. Dos de los dispositivos validados para la estimación indirecta de la presión arterial central. (A) SphygmoCorTM (AtCor MedicalLtd, West Ride, Australia); (B) Pulse Pen ${ }^{\mathrm{TM}}$ (Dia Tecne s.r.l., Milán, Italia)

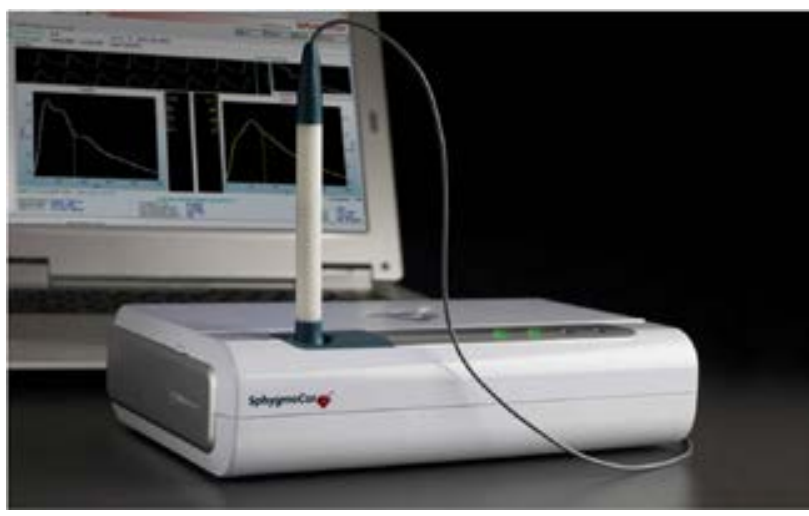

onda de pulso durante 24 horas (Figura 2).

\section{Limitaciones de la PA periférica}

La técnica habitual de medición de la PA periférica apenas se ha modificado en el último siglo y atiende únicamente a los picos máximo y mínimo de presión (PA sistólica y diastólica respectivamente), sin atender al resto de la onda de pulso. La sencillez de la técnica y su accesibilidad sin duda han permitido su amplia difusión. Sin embargo, son varias las limitaciones que presenta, entre las cuales destaca: 1) el denominado "efecto ó reacción de bata blanca", esto es la elevación transitoria de PA durante la medición realizada por personal sanitario; 2) la notable variabilidad de la PA que minimiza la reproducibilidad de una única lectura de PA y 3) numerosas fuentes de error internas (del sujeto) o externas (del proceso) que pueden modificar el resultado de la lectura (ej. perímetro braquial, arritmias, colocación del manguito o tamaño del brazalete). Nadie cuestiona el enorme valor desde el punto de vista epidemiológico que la PA clínica posee y en base a esta técnica y estos resultados basamos la mayor parte de nuestras decisiones en la práctica clínica diaria. Pero el valor predictivo de la PA clínica es limitado en la evaluación individual ${ }^{9}$.

Diversos estudios han mostrado una mayor correlación de la PA central (en comparación con la PA periférica) y diversos marcadores de lesión orgánica subclínica como la hipertrofia ventricular izquierda ${ }^{10} 0$ el espesor íntima media ${ }^{11}$. Además, la PA central ha demostrado un mayor valor predictor de eventos cardiovasculares que la PA periférica ${ }^{11,12}$. Finalmente, distintos agentes antihipertensivos a igualdad de logros tensionales de PA periférica consiguen diferentes logros en cuanto a reducción de eventos cardiovasculares coincidiendo con diferente reducción en las cifras de PA central ${ }^{13,14}$. En este sentido, destaca la controversia generada en torno a los resultados del estudio Heart Outcomes Prevention Evaluation (HOPE), en los que el tratamiento con ramipril conseguía no solo reducir la hipertrofia ventricular izquierda sino disminuir el riesgo de eventos cardiovasculares mayores (infarto

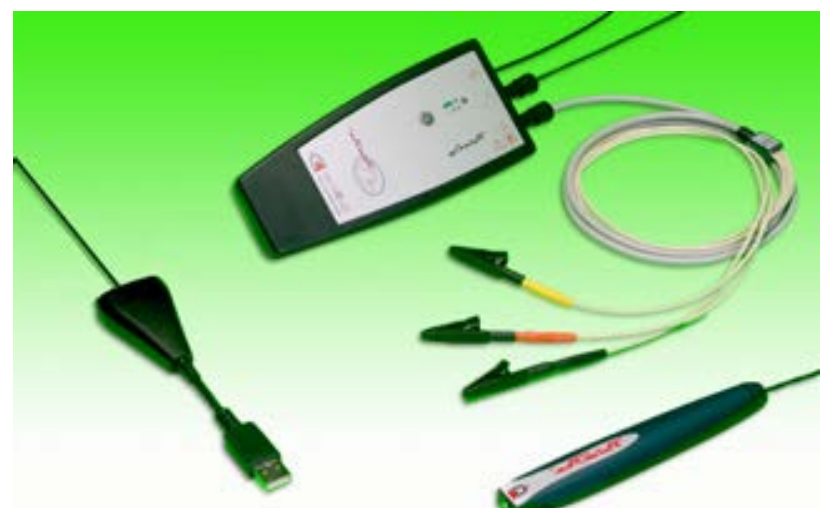

agudo de miocardio, ictus o insuficiencia cardíaca congestiva) y todo ello independientemente de la reducción de PA clínica alcanzada. Los propios autores acabaron finalmente reconociendo la probable relación del efecto vasodilatador del ramipril, el incremento en la distensibilidad arterial y el descenso en la reflexión de la onda como factores determinantes de los resultados obtenidos sin detrimento de otras posibles justificaciones (mecanismos neurohormonales o administración temporalizada de la medicación antihipertensiva) $^{15}$.

\section{Tonometría por aplanamiento}

La TA es un método sencillo, reproducible incluso en manos de personal no entrenado, validado e incruento para el análisis de la onda de pulso y la estimación de la PA central ${ }^{16}$.

Aplicando un sensor de presión perpendicularmente sobre la pared arterial podemos conocer las variaciones de presión en cada fase del pulso. Debido a la peculiar anatomía de los vasos sanguíneos, con un corte transversal circunferencial, necesitamos "aplanar" ligeramente la pared vascular para asegurarnos un íntimo contacto entre el sensor de presión (tonómetro) y la pared arterial (Figura 3).

Figura 3. Una vez que se localiza (palpación) el punto de máxima pulsación arterial, el sensor se ubica sobre la piel a ese nivel, presionando contra las estructuras sólidas subyacentes.
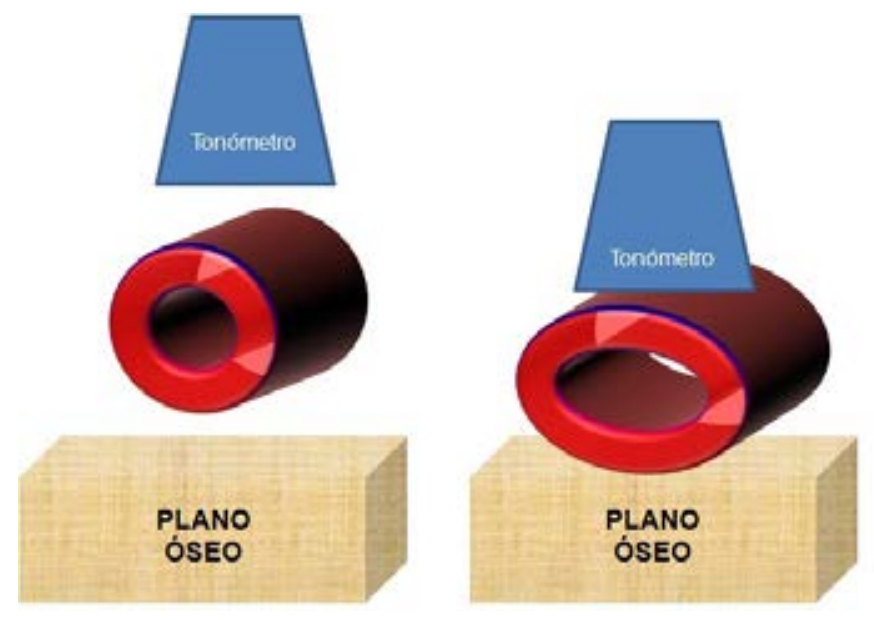
Si bien los registros obtenidos de la tonometría aplicada a arteria carótida o a la arteria subclavia se consideran iguales a los que se obtendrían a nivel central (no requieren aplicar funciones matemáticas para obtener la forma de onda central), no es menos cierto que presenta limitaciones relacionadas con las características del paciente $y / 0$ de las arterias (ej. sujetos obesos, ausencia de un plano óseo subyacente) $)^{17,18}$.

Por este motivo la arteria radial, que cuenta con una base sólida subyacente (apófisis estiloides del radio) nos permite obtener un registro fidedigno de la onda de pulso arterial. La señal obtenida debe tener una forma de onda reproducible y estable, los equipos más utilizados (p.ej. SphygmoCor®) requieren un registro de al menos 10 segundos y poseen sistemas de control de calidad del registro, en base a la homogeneidad de las ondas obtenidas (altura media y máxima de las ondas, pendiente de ascenso y valor basal o "presión diastólica").

El cálculo/estimación de los índices de presión central a partir de la PA periférica se realiza a través de un algoritmo matemático o "función de transferencia" validada y aprobada por la American Food and Drug Administration (FDA) para la estimación fidedigna de la PA central ${ }^{19-21}$. La validación de la función de transferencia braquial se llevó a cabo midiendo simultáneamente la PA aórtica y la PA radial mediante métodos invasivos ${ }^{20}$. Para ello, se tuvo en cuenta el fenómeno de amplificación periférica, por el cual las presiones arteriales diastólicas y medias en el ser humano apenas varían desde la raíz aórtica hasta la arterial radial (ya que no existe resistencia significativa en las grandes arterias de conducción) mientras que las presiones arteriales sistólicas y de pulso sí que adquieren mayor valor en arterias periféricas (son más estrechas y están más proximas a los puntos de bifurcación, donde la corriente sanguínea "colisiona" con la pared arterial y se genera un flujo retrógrado u "onda de reflexión")22.

De este modo, la onda de pulso obtenida en arteria radial mediante TA, asume la igualdad de la PA diastólica y media a nivel central y utiliza estos valores para estimar la PA sistólica y de pulso centrales.

\section{Reflexión de la onda de pulso}

Una parte esencial en la forma de la onda de pulso es la onda de reflexión. Cada segmento arterial desempeña dos funciones biomecánicas principales: por un lado permite que la sangre fluya hacia los tejidos ofreciendo mínima resistencia (función de "conducto") y por otra, amortigua la pulsatilidad generada principalmente por la actividad cardíaca (función "amortiguamiento"). Las propiedades viscoelásticas de la pared arterial (compliance) permite no solo almacenar/ transferir/disipar energía en cada pulsación sino mantener el flujo sanguíneo en el interior del circuito durante la diástole cardíaca, transformando el flujo sanguíneo pulsátil en un flujo más contínuo a nivel de tejidos periféricos.

Tras el incremento brusco de presión que se produce en el interior de la arteria como consecuencia de la sístole ventricular, una onda de presión de retorno (retrógrada) se genera por una doble vía: 1) la pared arterial se distiende y se incrementa la luz vascular para albergar la sobrecarga de volumen que llega con cada embolada ventricular, tras lo cual la pared arterial se retrae nuevamente y aparece una segunda onda de presión que se corresponde con la reflexión de la onda de pulso; 2) cuando el flujo de sangre "impacta" con arterias de menor calibre, bifurcaciones arteriales, placas de ateroma,... se constituye una onda de reflexión precoz (Figura 4).

Figura 4. Reflexión de la onda de pulso (área sombreada) como consecuencia de; (a) retracción de la pared vascular tras la sístole ventricular y (b) onda de flujo retrógrado procedente de la circulación periférica.
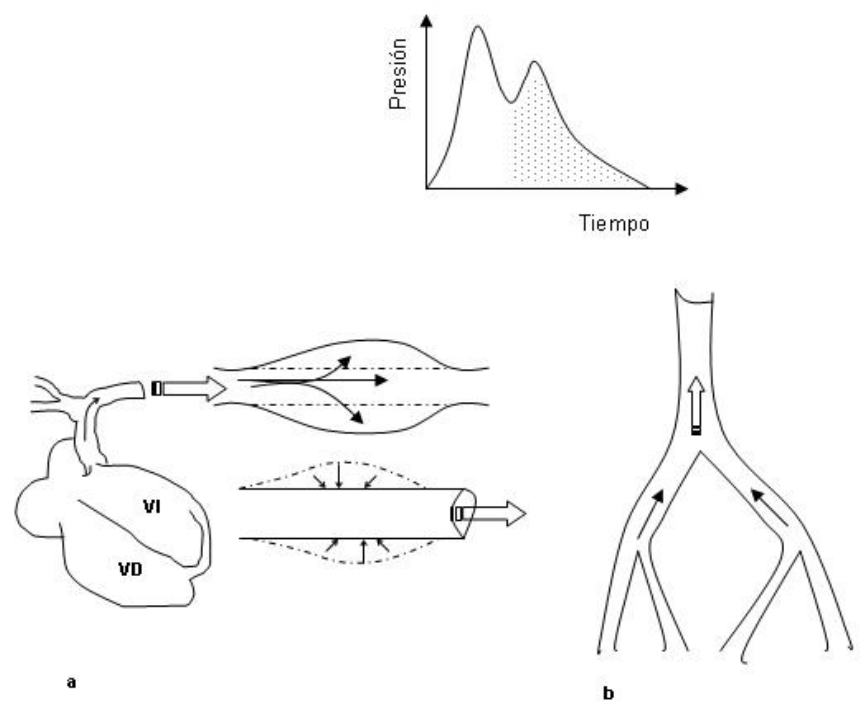

Con el envejecimiento fisiológico así como en el proceso arteriosclerótico, se origina un incremento de la rigidez vascular dando lugar a una onda de reflexión mayor y precoz. De este modo se sobrecarga el ventrículo izquierdo (contribuyendo a la larga a la aparición de disfunción sistólica/ diastólica, dilatación auricular, fibrilación auricular,...) y se reduce la presión durante la fase diastólica (período de perfusión coronaria), tal y como se representa en la Figura 5.

\section{Medición de la presión arterial central mediante tonometría}

La tonometría por aplanamiento aplicada en arterias superficiales como la arteria radial proporciona una onda de pulso prácticamente idéntica a la obtenida intraarterialmente ${ }^{22}$. Por este motivo, a la onda de pulso obtenida mediante TA en arterias periféricas (braquial, radial) se le otorga los va- 
Figura 5. (a) Onda tipo C (sujetos sanos, jóvenes) en la que la onda de reflexión se encuentra después de que se alcanze la presión sistólica máxima. (b) Onda tipo A (sujetos hipertensos, edad avanzada), las ondas reflejadas son precoces y contribuyen a aumentar la PA sistólica máxima.

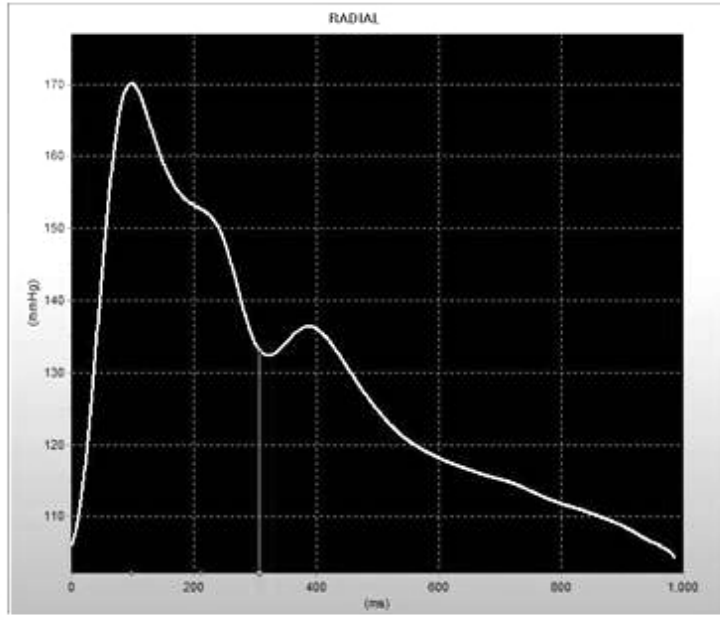

(a)

Iores de PA sistólica, PA diastólica, presión arterial media y presión de pulso obtenidos mediante esfigmomanometría convencional (Figura 6).

Una vez obtenida la onda de pulso periférica, ¿cómo se obtiene a partir de este registro, la onda de pulso aórtica 0 central? Existen dos métodos no invasivos y validados para obtener la presión arterial central: el método de calibración aplicado en arteria carótida común y la función de transferencia radial-aorta.

Tal y como comentamos anteriormente, en condiciones fisiológicas, a medida que la onda de pulso se propaga hacia las arterias periféricas, no solo se modifica la forma de la onda

Figura 6. Representación esquemática de la onda de pulso obtenida mediante tonometría en arteria radial. Los valores del pico máximo de presión (presión sistólica periférica) y del nadir de presión (presión diastólica periférica), son los obtenidos en arteria braquial a partir de un monitor de PA validado, disponible habitualmente en consulta. Del mismo modo, se calculan los valores de presión de pulso y presión arterial media.

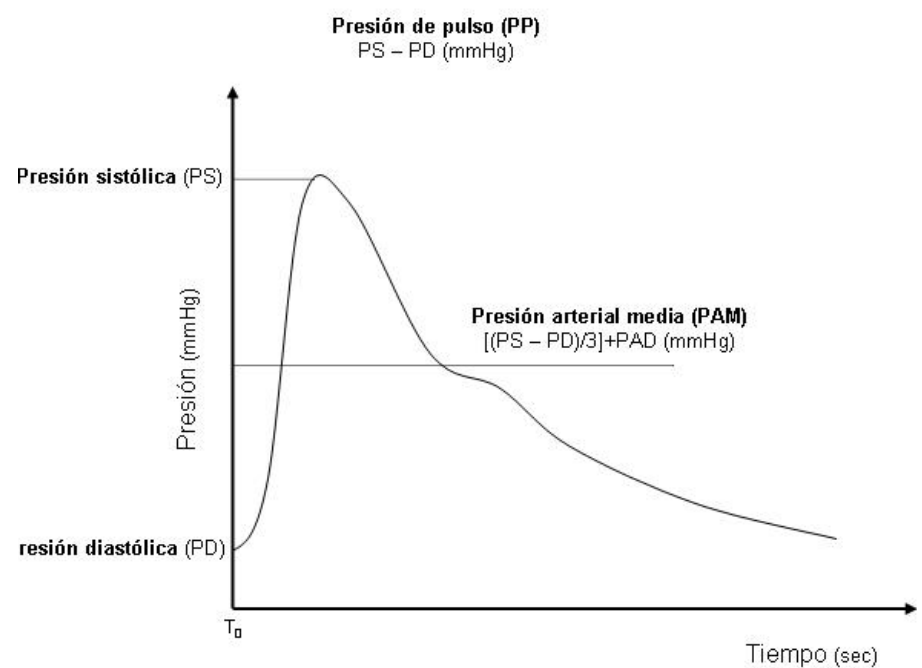

sino que aumentan la presión sistólica y la presión de pulso (fenómeno de amplificación) manteniéndose prácticamente sin cambios las presiones diastólica y media ${ }^{20}$. Partiendo de esta premisa y obteniendo la onda de pulso aórtico (central) aplicando el tonómetro sobre la arteria carótida común, a la onda de presión obtenida se le asigna;

- como valor de presión arterial diastólica aórtica (central): el valor de presión arterial diastólica en arteria braquial obtenida mediante esfigmomanometría convencional.

- como valor de presión arterial media aórtica (central): el valor de presión arterial media obtenida en arteria braquial mediante esfigmomanometría convencional, es decir: [(PA sistólica - PA diastólica)/3] + PA diastólica

- a partir de estos dos valores, se obtiene una escala que nos permite deducir el valor de presión para el pico máximo de la onda (presión sistólica central) y por ende, el valor de presión de pulso central.

Algunas de las limitaciones de este método de calibración aplicado en arteria carótida común, son las limitaciones vinculadas a las características del paciente (ej. obesos, cuello corto,...) lo que puede dificultar la obtención de una onda de pulso reproducible en arteria carótida, la ligera sobreestimación de la presión de pulso central (en comparación con la presión de pulso en aorta ascendente) o la contraindicación relativa en presencia de estenosis carotídeas (ej. placas de ateroma en carótidas) ${ }^{23}$.

El segundo método, la función de transferencia radial-aorta, es un método más sencillo que el primero y utiliza un algoritmo matemático que se obtuvo monitorizando intraluminalmente y de forma simultánea la onda de pulso en arteria radial y la onda de pulso en aorta ascendente, en una población de pacientes que iban a ser intervenidos quirúrgicamente ${ }^{20}$. Esta función de transferencia permite derivar 
la onda de pulso aórtica (central) a partir de un registro de onda de pulso radial (periférica) y ha sido validada posteriormente en nuevos ensayos clínicos lo que ha permitido su aprobación por parte de la Food and Drug Administration (FDA) para la estimación de la presión arterial central ${ }^{19,24}$.

Por su parte, algunas de las limitaciones de la función de transferencia son la aplicación de un método generalizado (obtenido a partir de una muestra poblacional) a una estimación individual (algo que no ocurría con el método de calibración) y sobre todo la utilización de valores de presión arterial sistólica periférica obtenidos en arteria braquial (con esfigmomanómetro) como equivalentes de presión arterial sistólica en arteria radial (con tonometría), subestimando el fenómeno de amplificación y que puede condicionar diferencias de hasta $7 \mathrm{mmHg}^{25}$.

\section{¿Cómo es la onda de pulso central en condiciones normales?}

Los picos máximos y mínimos de presión alcanzados por la onda de pulso periférica establecen los valores de presión sistólica y diastólica centrales respectivamente. La presión de pulso central (PPC) se estima, al igual que la presión de pulso periférica, por la diferencia entre la PA sistólica y la PA diastólica centrales.

El incremento de presión que supone la onda de reflexión sobre el pico de PA sistólica, es lo que se conoce como "incremento sistólico de presión" (en inglés, Augmentation pressure ; AP).

El cociente AP/PPc es lo que se conoce como "índice de aumento" 0 "índice de potenciación" (en inglés, "Augmentation index"; Alx) y se expresa como porcentaje. Habitualmente, y para establecer las estimaciones a condiciones estándar, éste índice se ajusta en función de la frecuencia cardíaca, equiparándose a un valor estándar de 75 latidos por minuto (Alx@75lpm). El Alx expresa, en términos relativos, la contribución de las reflexiones de onda a la PPc, y por lo tanto, es fiel reflejo del grado de rigidez arterial. La cuantificación del Alx no requiere que las ondas de presión se calibren (por ello, no es sensible a errores de calibración). La PA diastólica, la altura, la edad, la frecuencia cardíaca y la velocidad de onda de pulso son determinantes del Alx. Éste alcanza un valor estable alrededor de los 60 años (por lo que a partir de esa edad, perdería "sensibilidad"). La utilización del AP podría solucionar esa limitación.

La distancia que transcurre desde el inicio de la onda de pulso hasta el punto de inflexión donde comienza a descender, es lo que se conoce como "duración de la eyección" (DE). Por el contrario, el tiempo que transcurre desde el punto de inflexión de la onda hasta la finalización de la misma es lo que se denomina "índice de viabilidad subendocárdica" (SEVR) (Figura 7).
Figura 7. Representación esquemática de la onda de pulso central obtenida mediante tonometría por aplanamiento, junto con los distintos parámetros obtenidos a partir del análisis de la misma.

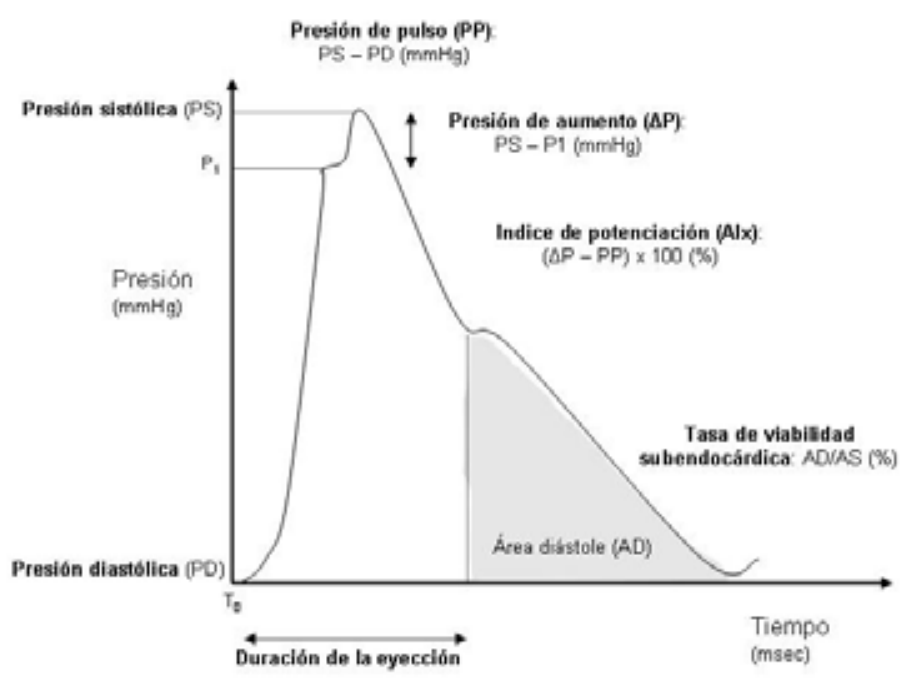

Los valores de PA central que establecen los rangos de normalidad según género y edad, se han ido delimitando a partir de estudios poblaciones, el más importante de ellos, sin duda el Anglo Cardiff Collaborative Trial26, que incluía a más de 4000 sujetos sanos. En líneas generales, la PA central normal, al igual que ocurre con la PA braquial, se incrementa con la edad, siendo ligeramente mayor en varones (al menos hasta la $6^{a}$ década de la vida) y alcanza un valor máximo de normalidad de $120 \mathrm{mmHg}+/-8 \mathrm{mmHg}$. Otros índices de la onda de pulso como la PPc, el AP y el Alx también se incrementan con la edad, pero alcanzan valores mayores en las mujeres y se debe considerar el inicio del manejo terapéutico en aquellos casos en los que la PA sistólica central sea mayor de $121 \mathrm{mmHg}$, la PPc mayor de $50 \mathrm{mmHg}$ (en varones > $45 \mathrm{mmHg}$ ) ó el AP/Alx mayor de 1 x desviación estándar de la media ${ }^{26,27}$.

Tal y como comentamos anteriormente, un aumento en la rigidez arterial condiciona una mayor y más precoz onda de reflexión lo que se traduce en un aumento en la presión sistólica y de pulso centrales, y una reducción de la PA diastólica central. Este incremento en la PA sistólica y de pulso centrales supone un estrés mecánico sobre la pared arterial (generando rotura de fibras de elastina, acumulación de colágeno, fibrosis, calcificación) además de una sobrecarga del ventrículo izquierdo. Esto contribuye a explicar la asociación entre un aumento de la rigidez arterial y el desarrollo de hipertrofia ventricular, lesiones en sustancia blanca cerebral, mayor riesgo de ictus y eventos coronarios ${ }^{28-30}$.

\section{Importancia de la onda de pulso en la estimación del riesgo cardiovascular. Valor pronóstico}

Diversos estudios longitudinales han demostrado el valor predictivo independiente del índice de aumento (Alx) y la PPC 
estimados mediante tonometría. En pacientes con enfermedad renal avanzada, ambos parámetros se correlacionaron de forma independiente con un incremento en la mortalidad total y mortalidad por causa cardiovascular ${ }^{31,32}$. También el Alx ha demostrado su valor predictivo de eventos cardiovasculares en pacientes con cardiopatía isquémica que iban a ser sometidos a intervención coronaria percutánea ${ }^{33}$. Pero sin duda, uno de los estudios más demostrativos del valor pronóstico de la PA central, la PPc y el Alx ha sido el estudio CAFÉ ${ }^{13}$, con un seguimiento de 3.4 años en población hipertensa de 2073 pacientes, demostrando además el mayor valor predictivo de la PA central en comparación con la PA periférica. Comparando dos estrategias de tratamiento antihipertensivo que consiguieron reducir la PA periférica de igual manera (atenolol amlodipino), aquellos pacientes con tratamiento con amlodipino lograban una reducción mayor de PA central y PPc al tiempo que alcanzaba una tasa menor de eventos cardiovasculares.

Un año después, el estudio STRONG ${ }^{10}$, demostraba cómo la PPc no sólo es un factor predictor de riesgo cardiovascular independiente sino que posee un mayor valor predictivo cuando se compara con la PP periférica (Hazard ratio; 1,15/10 mmHg 1,10/mmHg; $\left.X^{2}: 13,4 ; p<0,001\right)$. También en este estudio, aquellos pacientes con valores más altos de PA central y PPc, presentaban una mayor tasa de eventos cardiovasculares.

Otros estudios confirman esta aparente superioridad de la PA central frente a la PA periférica a la hora de predecir eventos cardiovasculares, incluso en poblaciones no seleccionadas 0 en sujetos de edad avanzada ${ }^{34}$.

Un revelador estudio, recientemente publicado, a partir de una cohorte de 1759 pacientes del proyecto Framingham, pone de manifiesto que aquellos pacientes normotensos con valores más altos del índice de aumento (Alx) tienen un riesgo incrementado de desarrollar hipertensión arterial (OR: 1,7; 95\% IC: 1,4-2) tras siete años de seguimiento del estudio $^{35}$. Estos datos sugieren que el incremento de rigidez vascular podría preceder al desarrollo de hipertensión arterial y no establecerse como consecuencia de la misma tal y como se ha venido postulando hasta la fecha.

También el incremento del Alx ha demostrado una correlación directamente proporcional con la escala de riesgo de Framingham ${ }^{36}$, así como con el riesgo de cardiopatía isquémica ó mortalidad de causa coronaria ${ }^{33}$.

En los últimos años, varios estudios han puesto de manifiesto el efecto deletéreo del incremento de la PA central en la incidencia de fibrilación auricular ${ }^{37}$, ó la asociación de PPc con la incidencia de hipertrofia ventricular izquierda, el espesor íntima media carotídeo o el incremento en el riesgo de enfermedad cerebrovascular ${ }^{38,39}$.

\section{Onda de pulso y tratamiento antihipertensivo}

Los diferentes fármacos antihipertensivos de los que disponemos en la farmacopea actual ejercen un mecanismo de acción basado en la vasodilatación arterial o en el descenso de la postcarga ventricular (reducción del volumen/latido y/o de la frecuencia cardíaca), pero no hay ninguno específicamente diseñado para mejorar las propiedades elásticas intrínsecas de la pared vascular. Sin embargo, el mecanismo de acción de los diferentes agentes antihipertensivos, los diferencia también a la hora de reducir los parámetros de PA central. Si hablábamos con anterioridad de cómo el estudio CAFE ${ }^{13}$, demostró mayor efecto del amlodipino en términos de reducción de PA sistólica y PP centrales, otros estudios hallaron del mismo modo mayor potencia antihipertensiva a nivel central comparando un agente bloqueante de los receptores de la angiotensina II (eprosartán) con ateno$10{ }^{40}, 0$ comparando una estrategia antihipertensiva basada en el tratamiento con IECAs frente a diuréticos tiazídicos ${ }^{41}$. El estudio REASON, comparando una estrategia basada en atenolol frente a otra con asociación perindopril/indapamida objetivó una mayor reducción de ésta última en términos de PA sistólica y de pulso centrales así como una mayor reducción de la hipertrofia ventricular izquierda comparado con el betabloqueante ${ }^{42}$.

También la reducción del Alx es mayor en pacientes tratados con ARA-II que aquellos que reciben atenolo| ${ }^{40}$, mientras que el tratamiento diurético no modifica de forma significativa este parámetro ${ }^{41}$.

En términos generales, los estudios publicados hasta la fecha ponen de manifiesto que el tratamiento con agentes diuréticos en monoterapia no presenta un beneficio adicional en términos de reducción de PA central más allá de la reducción de PA periférica. Algo parecido a lo que ocurre con el tratamiento betabloqueante que en algún estudio ha llegado incluso a asociarse a un incremento en la presión de pulso central (a pesar de alcanzar reducción en la presión de pulso periférica), si bien la práctica totalidad de los estudios publicados emplean únicamente atenolol como agente betabloqueante por lo que no disponemos de datos concluyentes acerca de los nuevos agentes betabloqueantes con propiedades vasodilatadoras. El incremento de Alx observado con el tratamiento betabloqueante se debe a su efecto bradicardizante que permite que la onda de reflexión se incorpore precozmente a la onda de presión sistólica, de tal modo que un descenso de 10 latidos/minuto, inducido por atenolol se asocia a un incremento del $4 \%$ en Al $x^{43}$.

Todos los estudios realizados con agentes dihidropiridínicos bloqueantes de canales de calcio objetivan un descenso de PA central aun mayor que el ejercido sobre la PA periférica, incluso en un escenario de bradicardia ${ }^{44}$. 
Los agentes bloqueantes del eje renina angiotensina aldosterona y en particular los inhibidores de la enzima conversora de la angiotensina (IECAs) han demostrado un efecto beneficioso en términos de reducción de rigidez arterial, en parte debido al efecto vasodilatador tras la disminución en los niveles de angiotensina II que conduce a la relajación en la musculatura lisa de la pared vascular ${ }^{43}$. Si bien, otros mecanismos independientes de la reducción de angiotensina II, podrían contribuir en este sentido, tales como la reducción de marcadores inflamatorios y niveles de estrés oxidativo 0 el remodelado de las fibras de colágeno/elastina ${ }^{44}$. Hasta la fecha son todavía escasos los estudios que puedan afirmar con rotundidad un efecto similar en el caso de los agentes antagonistas de los receptores de la angiotensina II (ARA-II), aunque la evidencia reportada parece apuntar en esa misma dirección $n^{45}$.

Se deduce por tanto que el empleo de agentes antihipertensivos con propiedades vasodilatadoras (calcioantagonistas dihidropiridínicos, IECAs o ARA-II) presentan un perfil más favorable en términos de reducción de PA central, en probable relación con una reducción de la amplitud y/o un retraso en la onda de reflexión.

\section{Conclusiones}

Existen, hoy por hoy, numerosas evidencias que apuntan al valor de la PA central como factor pronóstico de riesgo cardiovascular. Aunque muchos de estos estudios reclutan un número limitado de pacientes ó están diseñados de forma heterogénea (diferentes end-points, diferentes plazos de ejecución, ...), limitando por el momento su generalización, lo cierto es que no disponemos en la actualidad de objetivos de PA central para el manejo del paciente hipertenso, a diferencia de lo que ocurre con la PA periférica. Sin embargo, apunta a convertirse en una excelente herramienta, que junto con la PA periférica, puede ayudarnos a escoger una adecuada estrategia antihipertensiva.

Las guías de práctica clínica y los consensos de expertos sugieren realizar estudios no invasivos en sujetos asintomáticos para detectar y tratar la aterosclerosis en la etapa subclínica. La medición de la rigidez vascular mejora la categorización del riesgo cardiovascular y los cambios de la distensibilidad aórtica se han asociado a eventos cardiovasculares y calcificación aórtica ${ }^{46,47}$.

Existen importantes diferencias entre los diversos agentes antihipertensivos en lo que respecta a la reducción de PA central. Hasta la fecha, han sido los IECAs y los calcioantagonistas dihidropiridínicos (en monoterapia 0 en asociación) los que han demostrado un mayor efecto en este sentido en contraposición con los diuréticos o los betabloqueantes gracias entre otros mecanismos a la capacidad vasodilata- dora de los primeros unido a una reducción en la onda de reflexión.

Los sistemas de prevención cardiovascular basados en escalas de cálculo del riesgo presentan limitaciones para cuantificar el riesgo de un sujeto ya que predicen aterosclerosis pero no identifican a aquellos sujetos que desarrollarán la enfermedad. La tonometría por aplanamiento es una técnica sencilla, incruenta y reproducible de obtener la PA central y estimar el grado de rigidez vascular mediante el análisis de la onda de pulso. Las principales limitaciones hoy por hoy, para su aplicación de forma generalizada son su elevado coste así como la ausencia de grandes estudios de intervención capaces de posicionar alguna de las variables mencionadas como un objetivo terapéutico. Sin embargo, los estudios publicados hasta la fecha y otros muchos en curso nos obligan a prestar especial atención a una herramienta que podría optimizar el abordaje diagnóstico y terapéutico del paciente con hipertensión arterial y riesgo cardiovascular.

\section{Bibliografía}

1. O'Rourke MF, Seward JB. Central arterial pressure and arterial pressure pulse: new views entering the second century after Korotkov. Mayo ClinProc. 2006; 81: 10571068.

2. Nichols W, O’Rourke MF. EdsMcDonalds. Blood flow in arteries: theoretical, experimental and clinical principles. $5^{\text {th }}$ edition. Edward Arnold: London, 2005.

3. Agabiti-Rosei E, Mancia G, O’Rourke MF, Roman MJ, Safar ME, Sumulyan $\mathrm{H}$ et al. Central blood pressure measurements and antihypertensive therapy: a consensus document. Hypertension 2007; 50 (1): 154-160.

4. 4. Mancia G., De Backer G., Dominiczak A., Cifkova R., Fagard R., Germano G., Grassi G., Heagerty AM., Kjeldsen SE., Laurent S., Narkiewicz K., Ruilope L., Rynkiewicz A., Schmieder RE., StrujkerBoudier HA., Zanchetti A. 2007 Guidelines for the management of arterial hypertension: The Task Force for the Management of Arterial Hypertension of the European Society of Hypertension (ESH) and the European Society of Cardiology (ESC). J Hypertens 2007; 25: 1105-1187.

5. Snellen HA. E.J. Marey and Cardiology: Physiologist and Pioneer of technology (1830-1904). Rotterdam. TheNetherlands: KooykerScientificPublications; 1980.

6. Forssman W. Die Sondierung des rechtenHerzens. KlinischeWochenschrift. 1929; 8: 2085-87.

7. Kroeker EJ, Wood EH. Comparison and simultaneously recorded central and peripheral arterial pressure pulses during rest, exercise and tilted position in man. Circ Res. 1955; 3 (6): 623-632.

8. Pressman GL and Newgard PM. A transducer for the continuous external measurement of arterial blood pressure. IEEE Trans Biomed Eng. 1963; 10: 73-81.

9. Pickering TG, Shimbo D., Hass D. Ambulatory blood pressure monitoring. N Eng. J Med. 2006; 354: 2368-74.

10. Roma MJ, Okin PM, Kizer JR, Lee ET, Howard BV, Devereux RB. Relations of central and brachial blood pressure to left ventricular hypertrophy and geometry: The Strong Heart Study. J. of Hypertens 2010; 28 (2): 384-388.

11. Roman MJ, Devereux RB, Kizer JR, Lee ET, Galloway JM, Ali T et al. Central pressure more strongly relates to vascular disease and outcome than does brachial pressure: The Strong Heart Study. Hypertension 2007; 50 (1): 197-203.

12. Safar ME, Blacher J, Pannier B et al. Central pulse pressure and mortality in endstage renal disease. Hypertension 2002; 39 (3): 735-738.

13. Williams B, Lacy PS, Thom SM et al. Different impact of blood pressure-lowering drugs on central aortic pressure and clinical outcomes: principal results of the Conduit Artery Function Evaluation (CAFE) study. Circulation 2006; 113 (9): 1213-1225.

14. London GM, Asmar RG, O'Rourke MF, Safar ME. Mechanism(s) of selective systolic blood pressure reduction after a low dose combination of perindopril/indapamide in hypertensive subjects: comparison with atenolol. J. Am CollCardiol. 2004; 43 (1): 92-99.

15. Nichols W, Schuler B and O'Rourke MF. Ramipril and Cardiovascular Risk Reduction. Circulation 2002; 105: 194-195.

16. Crilly M., Coch C., Bruce M., Clarck $H$ and Williams D. Indices of cardiovascular function derived from peripheral pulse wave analysis using radial applanation tonometry: a measurement repeatability study. Vascular Medicine 2007; 12: 189-197. 
17. Laurent S., Cockroft J., Van Bortel L., Boutouyrie P., Giannattasio C., Hayoz D. et al Expert consensus document on arterial stiffness: methodological issues and clinical applications. Eur Heart J 2006; 2588-605.

18. Laurent S., Boutouyrie P., Asmar R., Gautier I., Laloux B., Guize L. et al. Aortic stiffness is an independent predictor of all-cause and cardiovascular mortality in hypertensive patients. Hypertension 2001; 37: 1236-41.

19. Chen $\mathrm{CH}$, Nevo E, Fetics B et al. Estimation of central aortic pressure waveform by mathematical transformation of radial tonometry pressure: validation of generalized transfer function. Circulation. 1997; 95 (7): 1827-1836.

20. Karamanoglu M, O'Rourke MF, Avolio AP, Kelly RP. An analysis of the relationship between central aortic and peripheral upper limb pressure waves in man. EurHeart J. 1993; 14 (2): 160-167.

21. Pauca AL, O'Rourke MF, Kon ND. Prospective evaluation of a method for estimating ascending aortic pressure from the radial artery pressure waveform. Hypertension. 2001; 38 (4): 932-937.

22. Kelly R., Hayward C., Ganis J., Daley J., Avolio A., O’Rourcke M. Non-invasive registration of the arterial pressure pulse waveform using high-fidelity applanation tonometry. J. Vasc Med Biol 1989; 3: 142-9

23. Van Bortel LM., Balkestein EJ., Van der Heijden-Spek JJ., Vanmolkot FH., Staessen JA., Kragten JA. et al. Non invasive assessment of local arterial pulse pressure: comparison of aplanation tonometry and echo-tracking. J Hypertens 2001; 19: 1037-44.

24. Gallagher D., Adji A. and O'Rourcke M. Validation of the transfer function technicque for generating central from peripheral upper limb pressure waveform. Am J. of Hypert. 2004; 17: 1059-67.

25. Pauca AL., Wallenhaupt SL., Kon ND., Tucker WY. Does radial artery pressure accurately reflect aortic pressure? Chest 1992; 102: 1193-8.

26. McEniery CM, Yasmin, Hall IR, Qasem A, Wilkinson IB, Cockcroft JR. Normal vascular aging: diferential effects on wave reflection and aortic pulse wave velocity: The Anglo Cardiff Collaborative Trial (ACCT). J. Am. CollCardiol. 2005; 46 (9): 1753-1760.

27. McEniery CM, Yasmin, McDonell B et al. Central pressure: variability and impact of cardiovascular risk factors: The Anglo Cardiff Collaborative Trial II. Hypertension 2008; 51 (6): 1476-1482.

28. Boutourye P, Laurent S., Girerd X., Beck L., Abergel E., Safar M. Common carotid artery distensibility and patterns of left ventricular hypertrophy in hypertensive patients. Hypertension 1995; 25: 651-9.

29. Laurent S, Boutourye P., Lacolley P. Structural and genetic bases of arterial stiffness. Hypertension 2005;45: 1055-55.

30. Liao D., Cooper L., Cai J., Toole J., Bryan N., Burke G., Shahar E., Nieto J., Mosley T and Heiss $G$. The prevalence and severity of white matter lesions, their relationship with age, ethnicity, gender and cardiovascular disease risk factors: the ARIC study. Neuroepidemiol.1997; 16: 149-162.

31. London GM., Blacher J., Pannier B., Guerin AP., Marchais SJ. Safar ME. Arterial wave reflections and survival in end-stage renal failure. Hypertension 2001;38: 434-38.

32. Safar ME., Blacher J., Pannier B., Guerin A., Marchais SJ., Guyonvarc'h PM., London GM. Central pulse pressure and mortality in end stage renal disease. Hypertension 2002; 39: 735-738.

33. Weber T. Auer J., O'Rourke MF., Kvas E., Lassning E., Lamm G., Stark N., Rammer M., Eber B. Increased arterial wave reflections predict severe cardiovascular event in patients undergoing percutaneous coronary interventions. Eur Heart J. 2005;26: 2657-63.
34. Pini R., Cavallini MC., Palmieri V et al. Central but not brachial blood pressure predicts cardiovascular events in an unselected geriatric population: the ICARe Dicomano Study. J Am Coll Cardiol. 2008; 51: 2432-39.

35. Kaess BM., Rovy J., Larson MG., Hambury NH., Vita JA., Levy D., Benjan ES., Vasan RS., Mitchell GF. Aortic stiffness, blood pressure progression, and incident hypertension. JAMA 2012. Sept 5; 308 (9): 875-81.

36. Van Trijp MJ, Uiterwaal CS, Bos WJ, Oren A Grobbee DE, Bots ML. Non invasive arterial measurements of vascular damage in healthly young adults: relation to coronary heart disease risk. Ann Epidemiol. 2006; 16 (2): 71-77.

37. Tsang TS, Verzosa GC, Barnes ME et al. Abstract 3163: central pulse pressure as a robust predictor of first atrial fibrillation: Study of Atrial Fibrilation in High Risk Elderly (SAFFIHRE). Circulation. 2008; 118: S_1106

38. Wohlfahrt P., Wichterle D., Seidlerova J., Filipovsky J., Bruthans J., Adamkova V. and Cifkova R. Relation of central and brachial blood pressure to left ventricula hyperthophy. The Czech Post-MONICA Study. Journal of human Hypertension 2012; 26: $14-19$.

39. De Silva D., Woon F.P., Chen C., Chang H.M., Kingwell B., Cameron J., Wong M.C. Profiule and association of ventral pulse wave velocity and central pulse pressure among ischemic stroke patients. European Journal of Neurology 2008; 15 (2): 196 198.

40. Klingbeil AU. Jhon S, Schneider MP, Jacobi J, Weidinger G, Scmieder RE. AT1-receptor blockade improves augmentation index: a double-blind, randomized, controlled study. J. Hypertens. 2002; 20 (12): 2423-2428.

41. Jiang XJ, O'Rourke MF, Zhang YQ, He XY, Liu LS. Superior effect of angiotensinconverting enzyme inhibitor over a diuretic for reducing aortic systolic pressure. J Hypertens. 2007; 25 (5): 1095-1099.

42. De Luca N. Asmar RG., London GM., O'Rourcke MF. REASON Project investigators. Selective reduction of cardiac mass and central blood pressure on low-dose combination perindopril/indapamide in hypertensive subjects. J Hypertens 2004; 22 : 1623-1630.

43. Protogerou AD., Papaioannou TG., Lekakis JP., Blacher J., Safar ME. The effect of antihypertensive drugs on central blood pressure beyond peripheral blood pressure. Part I: (Patho)-physiology, rationale and perspective on blood pressure amplification. Curr Pharm Des. 2009; 15: 267-71.

44. London GM., Pannier B., Vicaut E. et al. Antihypertensive effects and arterial haemodynamic alterations during angiotensin converting enzyme inhibition. J Hypertens. 1996; 14: 1139-46.

45. Matsui Y., Eguchi K., O'Rourcke MF et al. Differential effects between a calcium channel blocker and a diuretic when used in combination with angiotensin II receptor blocker on central aortic pressure in hypertensive patients. Hypertension 2009; 54 : 716-23.

46. Haluska BA, Jeffries L, Carlier S, Marwick TH. Measurement of arterial distensibility and compliance to assess prognosis. Atherosclerosis 2010; 209 (2): 474-480.

47. Blaha MJ, Budoff MJ, Rivera JJ, Katz R, O'Leary DH, Polak JF et al. Relationship of carotid distensibility and toracic aorta calcification: multi-ethnic study of atherosclerosis. Hypertension 2009;54: 1408-1415. 